\title{
QUISIERA QUE ME EXPLICARA EL SUBJUNTIVO. ACTITUDES Y PERCEPCIONES DE HABLANTES DE HERENCIA DE ESPAÑOL EN EL CONTEXTO DE ESTUDIOS EN EL EXTRANJERO
}

\author{
Quisiera que me explicara el subjuntivo. Attitudes and perceptions of Spanish \\ Heritage Speakers in Study Abroad Context
}

\author{
Munia Cabal Jiménez ${ }^{1}$
}

\begin{abstract}
RESUMEN
Este estudio analiza las percepciones de hablantes de herencia acerca de su propia variedad y sus actitudes y percepciones en el contexto de estudios en el exterior. Se analizan y contrastan las percepciones de hablantes de herencia que participan en un programa en España, por un período de tres meses, versus un grupo que participa en un programa en Costa Rica por un mes. El objetivo de este estudio es determinar, si después de haber participado en programas de estudio de lengua española en estos países, la experiencia impacta o modifica la percepción y comprensión sobre su español de herencia y, si diverge, en cuál parámetro. Los participantes son todos de la región del Medio Oeste, Estados Unidos (particularmente de Illinois), nacidos en los Estados Unidos y mayormente de origen mexicano. Los datos se recogen a través de un cuestionario aplicado via Qualtrics o personal que contiene veinticuatro preguntas en torno al perfil y bagaje del estudiante antes de dirigirse al programa de estudios en el extranjero, percepciones y reacciones durante los exámenes de ubicación escritos y orales, y su propia valoración después del programa. Las preguntas se circunscriben al ámbito académico en el país de inmersión.
\end{abstract}

Palabras clave: estudios en el extranjero, hablantes de herencia, español, actitudes, percepciones.

\begin{abstract}
This study analizes the perceptions of heritage speakers of Spanish regarding their own language variety and their attitudes and perceptions about studying abroad in Spanish-speaking countries. The perceptions of heritage speakers that participate in a program in Spain for three months, versus heritage speakers that participate in a program in Costa Rica for one month, are analyzed and compared. The goal of this study is to determine if after having participated in study abroad programs focused on Spanish language in these countries, the experience impacts and modifies the perception and understanding on their own heritage speaker variety. If different, the study try to determine in which parameter. The students that participated in the survey are all heritage speakers of Spanish from the USA's Midwest region (particularly from Illinois), born in the US and mostly of Mexican descent. Data were gathered via survey applied virtually via Qualtrics or personally, with twentyfour questions about the background of the students before going abroad, perceptions and reactions while taking written and oral placement tests, and their own perception after the program. The questions are focused on the academic experiences in each country of immersion.
\end{abstract}

Key Words: study abroad, heritage speakers, Spanish, attitudes, perceptions.

\footnotetext{
${ }^{1}$ Western Illinois University. Doctora en Lingüística Hispánica. Profesora Asistente. Departamento de Lengua y Literatura Extranjeras. Estados Unidos.

Correo: m-cabal-jimenez@,wiu.edu
}

Recepción: 06-12-18 Aceptación: 09-12-18 


\section{Introducción}

Con el incremento de hablantes de herencia de español (en adelante, HH) que ingresan a instituciones de educación superior en los Estados Unidos, la experiencia de ir a estudiar al extranjero para este grupo (particularmente para aquellos que requieren un nivel de proficiencia de otra lengua en sus futuras carreras profesionales) se ve como una forma en la que no solo se satisface un requisito de graduación, sino que también se valora como una posibilidad a través de la cual pueden "mejorar" su español, el cual se caracteriza por ser de naturaleza coloquial y con muy poca o ninguna experiencia académica. Tener la oportunidad de estudiar en el extranjero como parte de un programa de desarrollo en la lengua de herencia es vital porque este tipo de programas brindan posibilidades que no se pueden ofrecer en las universidades (Delany-Barmann et al., 2012).

El presente estudio presenta los resultados de un estudio que analiza las actitudes y percepciones de $\mathrm{HH}$ de español en el contexto de estudios en el extranjero. Como primer paso, sería importante recordar la clásica definición de Guadalupe Valdés, de qué se entiende por $\mathrm{HH}$ :

Los educadores de lenguas extranjeras usan el término para referirse al estudiante de lengua que crece en un hogar donde se habla una lengua diferente del inglés, que habla o apenas entiende la lengua de herencia y quien es, hasta cierto grado, bilingüe en esa lengua y en inglés (Valdés, 2001, p. 2). ${ }^{2}$

Con el creciente interés mostrado tanto por hablantes de herencia como por estudiantes de español como segunda lengua en participar en programas de estudios en el exterior (en adelante, PEE), se ha dado un renovado interés por parte de la comunidad académica por investigar los beneficios y limitaciones de estas experiencias. En ese sentido, Marijuan y Sanz (2018) indican que la investigación en el área de PEE se beneficiaría significativamente al investigar sobre la experiencia de inmersión de $\mathrm{HH}$, área a la que se le ha dedicado muy poca atención (Marijuan y Sanz, 2018, p. 187).

\footnotetext{
${ }^{2}$ A menos que se indique lo contrario, todas las traducciones de las citas originales en inglés son propias de la autora: "Foreign language educators use the term to refer to a language student who is raised in a home where a non-English language is spoken, who speaks or at least understands the language, and who is to some degree bilingual in that language and in English" (Valdés, 2001, p. 2).
} 
De acuerdo con el reporte conocido como Open Doors publicado por el Institute of International Education (IIE, por sus siglas en inglés) del año $2017,{ }^{3}$ la participación de estudiantes estadounidenses aumentó un 4 \% en comparación con el año anterior. Este mismo reporte también apunta que el número de personas que estudiaron en Latinoamérica y en la región caribeña incrementó hasta un 5.6 \%, convirtiendo a esta región en la más visitada después de Europa. Los dos países hispanohablantes que se encuentran entre los diez más visitados como destinos de estudio en el extranjero, según la lista elaborada por Open Doors, son, precisamente, España (tercer lugar) y Costa Rica (noveno lugar). ${ }^{4}$ En términos demográficos, de todos los estudiantes que estudian en el extranjero, de acuerdo con los criterios (estadounidenses) de raza e identidad, los hispanos o latinos constituyen el segundo grupo más grande después de los estudiantes caucásicos. Mientras que los estudiantes anglosajones constituyen el $71.6 \%$ en esa misma categoría, los latinos constituyen el $9.7 \%$ (Institute of International Education, 2017; Cubillos e Ilvento, 2013). El siguiente cuadro contrasta el número de estudiantes que participan en este tipo de programas de acuerdo con la raza y la etnicidad.

\section{Cuadro 1. Raza/Etnicidad de estudiantes estadounidenses en el extranjero}

\begin{tabular}{|c|c|c|c|c|c|}
\hline $\begin{array}{l}\text { Raza/Etnicidad de } \\
\text { estudiantes } \\
\text { estadounidenses }\end{array}$ & $2003-2004$ & $2008-2009$ & 2013-2014 & $2014-2015$ & $2015-2016$ \\
\hline Anglosajones & $83.7 \%$ & $80.5 \%$ & $74.3 \%$ & $72.9 \%$ & $71.6 \%$ \\
\hline Hispanos o Latino(a)(s) & $5.0 \%$ & $6.0 \%$ & $8.3 \%$ & $8.8 \%$ & $9.7 \%$ \\
\hline
\end{tabular}

\footnotetext{
${ }^{3}$ Este es el último reporte disponible de Open Doors al momento de escribirse este artículo.

${ }^{4}$ España tiene 46659302 millones de habitantes y múltiples instituciones de educación superior: 52 universidades públicas y 32 privadas y 157 academias de español como segunda lengua con acreditación del Instituto Cervantes. España, además, cuenta con el Instituto Cervantes, en España y alrededor del mundo, donde es posible estudiar español. Costa Rica tiene solo 5003402 millones de habitantes y, considerablemente, un menor número de universidades e instituciones; 5 universidades públicas y 14 privadas (aunque hay más universidades privadas con un limitado número de carreras). De acuerdo con www.languageinternational.mx el número de academias privadas para estudiar español en Costa Rica asciende a 21, pero es probable que existan bastantes más.
} 


\begin{tabular}{lccccc}
\hline $\begin{array}{l}\text { Asiáticos o de las islas } \\
\text { del Pacífico }\end{array}$ & & $7.1 \%$ & $7.7 \%$ & $8.1 \%$ & $8.4 \%$ \\
\hline Afro-americanos & $3.4 \%$ & $4.2 \%$ & $5.6 \%$ & $5.6 \%$ & $5.9 \%$ \\
\hline Multiracial & $1.3 \%$ & $1.6 \%$ & $3.6 \%$ & $4.1 \%$ & $3.9 \%$ \\
\hline Nativos de Alaska & $0.5 \%$ & $0.5 \%$ & $0.5 \%$ & $0.5 \%$ & $0.5 \%$ \\
\hline $\begin{array}{l}\text { Total de estudiantes } \\
\text { estadounidenses en el }\end{array}$ & & & & & \\
\hline \\
extranjero
\end{tabular}

Fuente: Institute of International Education, 2017 (Fast-Facts).

De este cuadro, es importante llamar la atención en el hecho de que mientras el porcentaje de estudiantes anglosajones en el extranjero ha disminuido, todos los otros grupos han incrementado porcentualmente su participación. De todos los otros grupos minoritarios mostrados en el cuadro, los latinos muestran el más alto crecimiento porcentual $(0.9 \%)$, tendencia que concuerda con el patrón general de crecimiento demográfico en la población estadounidense. ${ }^{5}$

\section{Estado de la cuestión}

\subsection{Estudios previos: hablantes de herencia y estudios en el extranjero}

Existen muy pocos estudios que hayan analizado el impacto que ha tenido la experiencia de estudiar en el extranjero en $\mathrm{HH}$ de español y las percepciones que estos tienen sobre su propia variedad. La mayoría de estos estudios están enfocados en las actitudes de

\footnotetext{
${ }^{5}$ De acuerdo con la Oficina del Censo de los Estados Unidos, para el 1 de julio de 2016, la población latina de los EE.UU. constituía el $17.8 \%$ de total de la población estadounidense. Solamente entre 2015 y 2016 hubo un incremento del $2 \%$ a través del país. Para el año 2060, de acuerdo con la misma fuente, la proyección es que, aproximadamente, un $28.6 \%$ de la población estadounidense estará constituida por latinos, unos 119000000 de personas (US Census Bureau, Population Division, 2017).
} 
hablantes de segunda lengua (Shiri, 2013; Weseley, 2012). La tesis doctoral de Moreno aborda algunos aspectos de etnicidad e identidad de hablantes de herencia de varias lenguas en Estados Unidos (alemán, coreano, chino, español, entre otras). Algunos estudios previos (Carrasco y Riegelhaupt, 2003; Davidson y Lekic, 2013) profundizan en aspectos identitarios de los hablantes de herencia en programas de estudios en el extranjero en Guanajuato (México) y en Nicaragua. Shively (2016) provee un panorama general de las motivaciones, identidad y desarrollo del lenguaje de los $\mathrm{HH}$ durante su estancia de estudios, con particular énfasis en países hispanohablantes. Su artículo apunta que las pocas investigaciones que versan sobre las motivaciones de los $\mathrm{HH}$ de estudiar en el extranjero están basadas en entrevistas, diarios y reportes personales en lugar de exámenes estandarizados para evaluar proficiencia. Para los propósitos de este estudio, el cual trata de recoger las autopercepciones acerca de por qué estudiar español en el extranjero, todos estos mecanismos de evaluación resultan apropiados por cuanto la meta de este trabajo no es determinar cuánto ha ganado el estudiante de herencia en términos de proficiencia, sino el determinar las actitudes y percepciones hacia su propia variante después de verse expuestos a otras variedades de español y a experiencias académicas relacionadas con el "aprendizaje” del español en instituciones educativas en el exterior.

Después de una revisión acuciosa de varios artículos y publicaciones, Shively (2016) establece las siguientes motivaciones por parte de los $\mathrm{HH}$ a la hora de decidirse por un programa de estudios de español en el extranjero, las cuales están divididas claramente en dos grupos: aquellos $\mathrm{HH}$ que desean estudiar en su país de origen y aquellos $\mathrm{HH}$ que desean estudiar en un país distinto al de sus ancestros. Entre las motivaciones para estudiar en el mismo país del cual procede su cultura de herencia se encuentran las que van desde una perspectiva más personal y familiar (profundizar en la cultura de la familia, el deseo de comunicarse con los parientes en la lengua de herencia), hasta aquellas de orden lingüístico (mejorar su español, verse inmersos en la lengua de herencia) e instrumental (mejorar las oportunidades de empleo en los Estados Unidos). La familiaridad o previos viajes al país de herencia pueden ser razones para querer buscar un destino distinto al del país de origen de la familia (2016, p. 264). Ninguno de estos estudios, por el momento, se refiere al componente ideológico sobre la lengua, esto es, sus propias actitudes y percepciones, de los hablantes de herencia en este tipo de programas. 


\subsection{Actitudes lingüísticas e ideologías del lenguaje}

Es un hecho bastante mencionado en la literatura que la percepción de los HH sobre su propia variedad es la de que hablan un "mal" español o un español "incorrecto":

La actitud hacia la propia variedad de lengua es otro factor que surge de las complejas circunstancias de los estudiantes de herencia. Algunos investigadores han observado que los estudiantes chicanos tienen a percibir su propio español como "malo" o “incorrecto [...]" (Said-Mohand, 201, p. 93). ${ }^{6}$

Después de examinar los diferentes acercamientos a partir de los cuales se puede analizar y entender la experiencia mexicoamericana en los Estados Unidos, a saber, la perspectiva lingüística, la sociolingüística y la lingüístico-crítica, Martínez identifica como componente fundamental la experiencia lingüística (Martínez, 2000, p. 7) que va más allá de la simple estructura de la lengua y convoca (incorpora) el despliegue y empleo de esta en situaciones sociales (con las potenciales implicaciones de su uso sociolingüístico y del posible posicionamiento sociopolítico que dicho empleo pueda acarrear).

De entre los varios elementos a los cuales se le debe prestar atención se encuentran los componentes de las actitudes lingüísticas y el de las ideologías sobre la lengua que -en la perspectiva de estudiantes de herencia de español de los EE. UU. que deseen estudiar en el extranjero- cobra una relevancia significativa.

Hay, en ese sentido, dos conceptos que conviene, sucintamente, presentar en la consideración y análisis de las actitudes y percepciones de y sobre los hablantes de herencia cuando estos escogen un programa de lengua española. Estos dos componentes, inextricablemente relacionados, son los conceptos de ideologías lingüísticas y de actitudes lingüisticas. Estrechamente relacionado está, desde luego, el concepto de percepción de sí mismos (lo que en otros términos en lingüística aplicada se conoce como self-efficacy o la percepción o idea que tiene el estudiante/hablante sobre sí mismo y su nivel de empleo y eficacia en el uso de la lengua (Cubillos e Ilvento, 2013).

\footnotetext{
6 “Attitude toward one's own language variety is another factor that stems from the complex circumstances of HL students. Some scholars observed that Chicano students tend to perceive their own Spanish as 'bad' or 'incorrect' [...]"' (Said-Mohand, 2011, p. 93).
} 
El concepto de ideologías lingüísticas puede definirse como el conjunto de ideas que tienen los individuos sobre las lenguas y la forma de hablar. Estas nociones, como conceptualiza Philips (2015), apuntan a que los comentarios que hacen las personas sobre las lenguas están determinados por "intereses económicos y políticos y por relaciones de dominación y subordinación” (Philips, 2015, p. 557).

Con frecuencia, estas ideologías lingüísticas han estado referidas no solo a la concepción de que las lenguas se corresponden con la forma en que estas se consideran legítimas en su práctica a partir de lo definido por otras instituciones o en relación con la constitución de estados y naciones. Esta relación entre la legitimidad de una lengua y una noción de estandarización, del conocimiento de la gramática, de una concepción estándar de la lengua, encuentra una expresión particular en lo que tradicionalmente se considera el deseo o la motivación -desde la institución- por parte del hablante de herencia al ir a un programa de estudios en el extranjero, con el fin de estudiar su lengua de herencia en un país hispanohablante. En el caso de comunidades de herencia en los Estados Unidos, se ha señalado la relevancia que tiene este concepto y sus diferentes expresiones en el contexto de las comunidades mexicano-americanas en el suroeste de los Estados Unidos. La interrelación de estos dos componentes (lengua e ideología) está fuertemente cargada de un significado social y político en este contexto, al indizar a los hablantes de español mexicano-americano como portadores de ciertos valores y creencias que los perfilan -indizan, otra vez- como usuarios de rasgos que divergen de aquellos considerados aceptables y cuyas diferencias y prácticas lingüísticas (como el code-switching) se constituyen en herramientas de subordinación lingüística. Como bien apunta Martínez (2000), las ideologías sobre las lenguas las tiene cada grupo (dominante o minoritario) sobre la lengua del otro. No se ha puesto en evidencia cómo impacta esta doble articulación sobre la lengua en el caso de hablantes de herencia que se inscriben en programas o cursos de español en países hispanohablantes.

El aspecto de la actitud lingüística es relevante en tanto que las actitudes sobre la lengua y sobre las variedades de esta lengua se extrapolan a la gente que las habla (Denison, 2004) y si bien desde la perspectiva de la lingüística popular (Jara, 2006) están basados en una serie de estereotipos sobre la lengua, las personas suelen establecer una correlación entre rasgos de la lengua y los de los grupos humanos que las hablan. Denison (2004) cita, a modo 
de ejemplo desde la perspectiva popular, cómo la gente percibe la rudeza de las consonantes alemanas como un reflejo de la frialdad alemana o el uso de variedades poco prestigiosas con la falta de inteligencia por parte de los hablantes que las emplean (2004, pp. 40-41).

\subsection{Programas de estudio en el extranjero y su impacto en el estudiante}

Estudios previos por Cubillos e Ilvento (2012) han demostrado que el impacto de la experiencia de estudios en el extranjero es diferente en estudiantes de L2 en relación con la mejoría adquirida en proficiencia lingüística: algunos obtienen un avance significativo, otros no muestran ningún desarrollo en términos lingüísticos; todos estos factores dependen de la cantidad de interacción y de la longitud del programa, entre otros elementos. Por lo tanto, es natural preguntarse si este tipo de experiencia puede, asimismo, tener un impacto en la percepción de los HH sobre su lenguaje o sobre la necesidad de mejorar su español de herencia, puesto que existe un común acuerdo en que esta experiencia -estudiar en otro paístiene un impacto en el nivel de conciencia cultural y en la motivación. Cubillos e Ilvento parten del concepto de autoeficacia (self-efficacy en inglés) para analizar cómo los estudiantes de español perciben su competencia (lingüística): "La autoeficacia se refiere al juicio de la gente sobre sus propias capacidades para completar con éxito una tarea" (Bandura, 1997, citado por Cubillos e Ilvento).

El concepto de autoeficacia ha sido empleado también como un mecanismo que permite predecir el éxito académico, pero otros factores tales como las experiencias previas de los estudiantes, la observación en cómo se desempeñan otros, así como el apoyo y la aprobación recibidas y los estados emocionales (por ejemplo, si el ser entendido por otros provee un sentido de autosuficiencia) también cuentan (2012, p. 497). Dado que el ambiente de estudios en el extranjero provee el contexto en el cual se persiguen tanto el éxito lingüístico como el académico, este último es también un factor en la consideración de las creencias y percepciones que los estudiantes de L2 tienen para consigo mismos. Es de esperarse que esta experiencia y el sentido de autosuficiencia tenga el mismo impacto en las creencias y percepciones de los hablantes de herencia.

Un último elemento mencionado en el estudio de Cubillo e Ilvento es el de que las comunicaciones no estructuradas con miembros de la comunidad meta (en el extranjero) 
también contribuyen con el nivel de la autoeficacia. Cabría, pues, preguntarse si los $\mathrm{HH}$ experimentan algún tipo de ganancia en este tipo de situaciones no estructuradas, puesto que estas situaciones espontáneas no constituyen para ellos un reto como para los estudiantes de L2. Por lo general, los $\mathrm{HH}$ ya se han visto expuestos a este tipo de situaciones comunicativas en la casa, en la escuela o en sus comunidades de herencia. Moreno (2009) al respecto expresa que existe una diferencia entre el tipo de experiencia vivida por el estudiante de L2 cuando va a estudiar a otro país y el estudiante de herencia. El discente de L2 descubre un ambiente y una cultura que le resultan "misteriosos" mientras que el de herencia no encuentra estas situaciones demasiado retadoras, debido a su cultura de herencia. Según Moreno "en este contexto, los estudiantes no están tan inmersos en la diferencia por cuanto ellos están explorando raíces recientes con las cuales todavía mantienen una conexión” (Moreno, 2009, pp. 1-2).

\subsection{Programas de estudio en el extranjero y el concepto de lengua promovido en universidades e institutos}

Al buscar en línea los tipos de cursos ofrecidos por institutos de lengua que están dirigidos a hablantes de herencia, también es posible acceder a las contribuciones metodológicas desde la perspectiva de ELE (Español como Lengua Extranjera) del Instituto Cervantes en Albuquerque. En esta última institución, es posible determinar que se concibe la enseñanza del español desde una perspectiva gramatical de corrección, debido al deseo del hablante de herencia de perfeccionar su lenguaje. El instituto Cervantes en Albuquerque, en su propuesta metodológica (Corros Mazón, 2005), estima que la validación de “dialectalismos poco prestigiosos" por parte de los hablantes de herencia se traduce en una actitud que percibe en la normalización una imposición que, de continuarse, podría prevenir el mantenimiento de una lengua estándar, sin especificar cuál variedad (estándar). En relación con la variedad estándar de español que el Instituto Cervantes promueve, Mar-Molinero y Paffey señalan la declaración hecha por el Instituto Cervantes de seleccionar la variedad de español del centro de España como la variedad por enseñar (es decir, la escogida como la “estándar”), posición también sustentada por la RAE. Estos autores establecen que: 
Resulta evidente tanto a partir de los objetivos establecidos por el Cervantes como por la RAE, la creencia de que una única variedad estándar de español es un medio neutral de participación social y de que la variación lingüística constituiría un impedimento para ello. Sostener que las normas detrás de un español pan-hispánico o global son "neutrales” o "anónimos” nos recuerda una vez más el peligro de no identificar la capacidad de acción [de la gente] cuando analizamos la difusión lingüística (Mar-Molinero y Paffey, 2011, p. 758). ${ }^{7}$

A esta red de promoción se suman una serie de institutos y universidades que, indirectamente, coadyuvan en la promoción de esta variedad; las academias miembros de la ASALE y, en el caso del español en los Estados Unidos, la presencia del Observatorio del Español en la Universidad de Harvard, que forma parte de la red del Instituto Cervantes. A esta implementación normativa se suma, como miembro activo de la ASALE, la Academia Norteamericana de la Lengua (ANLE) que, en su página web establece: "La ANLE tiene como misión el estudio, elaboración e implementación de las reglas normativas del español de los Estados Unidos de América" (www.anle.us).

Esta percepción globalizada sobre el español (Mar-Molinero y Paffey, 2011) también es considerada y estudiada en relación con el español de los Estados Unidos. En un estudio sobre actitudes lingüísticas se preguntó a hablantes de español de los Estados Unidos sobre su percepción acerca de cuáles y cuán similares son otras variedades de español con respecto al español hablado en los EE.UU. (Moreno Fernández, 2017). Los resultados reflejaron que los hispanohablantes no residentes en Estados Unidos no incluyen al español de los EE. UU. dentro de la colectividad hispana; por otro lado, los hablantes de español residentes en este país perciben que hay más distancia con el español de países hispanohablantes, (Latinoamérica y España) y aún más distancia con respecto a las variedades de español empleadas en países donde la lengua no es oficial (Moreno Fernández, 2017), una clasificación, pues, jerárquica. Así se afirma en la siguiente cita:

\footnotetext{
7 "Evident from both the RAE's and the Cervantes' stated objectives is the belief that a standard single variety of Spanish is a neutral medium of social participation and that language variation would be an impediment to this. Claiming that the norms behind pan-Hispanic or global Spanish are "neutral" or "anonymous" reminds us once again of the danger of not identifying agency when analyzing language spread" (Mar-Molinero and Paffey, 2011, p. 758).
} 
De este modo, el español estadounidense se aprecia como diferente del español de los países hispánicos, pero no se alinea con el utilizado por otros países en los que el español no es lengua oficial ni vehicular (Moreno Fernández, 2017, pp. 459-460).

Una conclusión importante de Moreno Fernández, relevante para el presente estudio, es el de que si bien las variedades de español como las de Latinoamérica son percibidas cognitivamente- como centrales, las variedades de español en países donde no es lengua oficial o mayoritaria son consideradas periféricas. Desde el punto de vista de los hispanohablantes no residentes en EE. UU., el español estadounidense es considerado también periférico. Para los hablantes de español residentes en los Estados Unidos, el español de este país constituye un área diferenciada (pero no periférica) dado que:

Si bien reconocen cierta distancia respecto de los primeros [hispanos no residentes en los EE. UU] no se alinean en absoluto con otros territorios bilingües, como Gibraltar o Guinea, en los que también se utiliza el español (2017, p. 461).

No obstante, en investigaciones realizadas hasta el momento en relación con $\mathrm{HH}$ en el contexto de estudios en el extranjero, no se observa una validación de la conciencia sociolingüística del estudiante sobre la variedad de herencia, es decir, no se enfatiza la importancia de la variación lingüística como ya ha sido sugerido (Villa, 1996 y 2002; Leeman y Serafini, 2016) o de la importancia del desarrollo de una conciencia crítica sobre el español de herencia (Leeman, 2005; Holguín, 2017). Muchos estudios han hecho referencia a la importancia de la identidad, a la elaboración de la identidad, pero aún existe un fuerte énfasis en la (así asumida) necesidad de proveer una evaluación del nivel de proficiencia del HH y del esperado mejoramiento que refleje de manera especular aquellas evaluaciones que se ejecutan para medir la proficiencia de estudiantes de L2 (Shively, 2016).

\section{Metodología}

Los estudiantes entrevistados realizaron sus estudios en una universidad localizada en la región del Medio Oeste de los Estados Unidos, específicamente, en la parte oeste de Illinois. Esta área se caracteriza por ser rural y su principal actividad consiste en la producción de maíz y soya. La comunidad inmigrante ha sido atraída a esta área por la posibilidad de trabajo que ofrecen las plantas empacadoras de carne (Albarracín, 2016, p. xxii). La 
población latina de la universidad viene en parte de la ciudad de Chicago, de algunos de sus suburbios, pero también de los pueblos más rurales que se encuentran cerca de la universidad, particularmente de aquellos que - como ya se mencionó- atraen a muchos trabajadores inmigrantes por las posibilidades de trabajo en la industria de empaque.

De acuerdo con Albarracín, los latinos no solamente presentan tendencias de movimiento demográfico hacia regiones diferentes de las del oeste de los EE.UU., sino que también han mostrado una tendencia clara de establecerse en áreas que no son urbanas, sino rurales. Este crecimiento demográfico en la región ha ido de un 33 \% en el año 2000 a un 104 \% en el 2010 en la región del Medio Oeste de los Estados Unidos (Albarracín, 2016). La mayoría de los estudiantes entrevistados para este estudio emplea una variedad de español de herencia que refleja rasgos de habla rural de México, no de español urbano. ${ }^{8}$ En términos de demografía sociolingüística, esta área se encuentra ubicada en la región del Medio Oeste de los Estados Unidos, que comprende un $9 \%$ de los latinos según los datos del censo de 2010 (Escobar y Potowski, 2015, p. 13). En esta universidad, el número total de estudiantes matriculados en el 2017 fue de 7,599 estudiantes (Otoño, 2017), de los cuales 994 son latinos $(10.7 \%)$, si se toma en cuenta tanto estudiantes de grado como de posgrado. ${ }^{9}$

\subsection{Recolección de los datos ${ }^{10}$}

Esta investigación empleó cuestionarios como instrumento para recoger los datos. También se obtuvo información de los diarios digitales escritos durante su estancia en el país de destino. Este instrumento ya ha sido aplicado con anterioridad para evaluar la experiencia de estudiantes de español como segunda lengua (Aguilar Stewart, 2010).

Esta investigación aboga por la incorporación de diarios digitales como una herramienta para asesorar la experiencia de estudios en el extranjero de muy variadas maneras " $[\ldots]$ los diarios digitales pueden proveer un espacio para entender la naturaleza

\footnotetext{
${ }^{8}$ Esta observación está basada en lo que se aprecia en los ensayos y trabajos de curso que la autora observa en clase, desde una perspectiva lingüística. Desde un punto de vista demográfico, esta afirmación se basa en los datos provistos por los estudiantes en el cuestionario.

${ }^{9}$ Western Illinois University. (2017-2018). Fact Book.

${ }^{10}$ Los datos fueron recogidos a través de un estudio llevado a cabo durante los semestres de otoño de los años 2015 y 2017, bajo el IRB \#3252 de la Comisión de Ética Científica de Western Illinois University (Office of Sponsored Projects, por sus siglas en inglés).
} 
ecológica (vivienda, clases, interacción con hablantes nativos) de los estudiantes en programas en el extranjero" (Aguilar Stewart, 2010, p. 154).

Los diarios digitales permitieron confirmar (o no) las percepciones observadas en el cuestionario.

\subsection{El cuestionario}

El cuestionario comprende 24 preguntas que inquieren sobre la información demográfica, la familia del estudiante, bagaje educativo, experiencia previa con el español en contextos académicos, factores que los motivan a participar en el programa de estudios en el extranjero y con preguntas de respuesta abierta (open-ended) sobre sus percepciones acerca de su nivel de conocimiento y cómo miden o califican su español. La encuesta fue administrada después de finalizado el programa de estudios. Los discentes fueron preseleccionados de entre todos los estudiantes que asistieron al programa, dado su perfil de HH. El análisis, los resultados y discusión se complementan con el material extraído de los diarios digitales y de las reflexiones finales. ${ }^{11}$ Solamente se pudo tener acceso a cuatro diarios digitales y a cuatro reflexiones finales de estudiantes que fueron a Costa Rica. ${ }^{12}$ Para consultar las preguntas del cuestionario, ver Apéndice I.

\subsection{Participantes}

Los informantes fueron diez estudiantes universitarios que participaron en dos programas de estudio en el extranjero enfocados en el estudio de la lengua española. De los diez, seis participaron en el programa de España (primavera de 2015 y de 2017) y otros cuatro participaron en el programa de Costa Rica (verano de 2015 y de 2017). La mayoría de los entrevistados son estudiantes de primera generación y provienen de familias que previamente no han tenido ningún tipo de exposición a la educación superior universitaria o a algún otro tipo de experiencia académica más allá de la secundaria o más allá de la primaria, en varios

\footnotetext{
${ }^{11}$ Las percepciones extraídas de los diarios digitales y de las reflexiones fueron primero producidas como parte de una tarea y no como parte de la encuesta.

${ }^{12}$ Solamente el programa en Costa Rica implementó los diarios digitales. El programa en España empleó diarios manuscritos y estos no pudieron conseguirse de parte de los estudiantes.
} 
casos. Ello excluye, en consecuencia, cualquier familiaridad con el proceso de estudiar en el extranjero.

\section{Análisis}

Al inicio del programa, los estudiantes realizaron exámenes de ubicación, tanto orales como escritos. Todos los estudiantes pudieron completar ambos exámenes. Seis fueron ubicados en nivel avanzado y cuatro fueron ubicados en nivel superior.

\subsection{Información demográfica sobre los estudiantes y perfil sociolingüístico}

De los diez estudiantes entrevistados, cuatro hombres y seis mujeres, ocho nacieron en los Estados Unidos y dos en sus países de origen y aprendieron español allí y luego vinieron a EE.UU. Cinco señalaron que el español es usado activamente en sus comunidades. No se reportó el uso del idioma exclusivamente en la casa, lo que significa que, para todos, el español se usa tanto en la casa como dentro y fuera del aula de clase, es decir, no hay diglosia. ${ }^{13}$ Es importante acotar algunos factores adicionales. Del $30 \%$ que tuvo algún acceso al español académico durante la primaria, el período de exposición oscila de los seis meses a tan solo un año. Del 50 \% que en secundaria recibió clases de español, el tipo de clase recibida también variaba, desde aquellas específicas de español como lengua de herencia hasta clases de español como L2 o de nivel avanzado. ${ }^{14}$ El $40 \%$ nunca había recibido instrucción en español hasta el momento de ingresar a la universidad.

\subsection{Resultados y discusión}

Los resultados cualitativos de la encuesta de actitudes aplicada a los estudiantes $(\mathrm{n}=$ 10) se articulan en torno a varios ejes, uno de ellos es la motivación para estudiar en el extranjero. Como se puede apreciar, independientemente del país de destino, la motivación mayor para asistir a estos programas es, efectivamente, mejorar el español y, en segundo

\footnotetext{
${ }^{13}$ Mejías y Anderson-Mejías (2003) reportan el uso no diglósico de las dos lenguas en el sur de Texas.

${ }^{14}$ Advanced placement, AP por sus siglas en inglés. Este tipo de curso es reconocido como curso de nivel universitario de primero o segundo año de carrera por el sistema educativo estadounidense.
} 
lugar, conocer un país (diferente) de habla hispana. En ese sentido, los resultados coinciden con tendencias ya articuladas en estudios previos (Shiri, 2013; Shively, 2016), tanto para estudiantes de L2 como para hablantes de herencia.

\subsubsection{Expectativas individuales}

Luego de solicitarles que escribieran las expectativas que abrigaban a nivel personal sobre su participación en el PEE, se les solicitó que las ordenaran por lugar de prioridad. El siguiente cuadro ilustra las expectativas de acuerdo con su importancia. El número indica el orden en que se escribieron (\#1, \#2, \#3) y el formato de la letra indica prioridad (uno en negrita, dos en cursiva y tres en subrayado). ${ }^{15}$

Cuadro 2. Expectativas individuales sobre la participación en el PEE

\begin{tabular}{|c|c|c|c|}
\hline País & $\# 1$ & $\# 2$ & $\# 3$ \\
\hline Costa Rica & Mejorar mi español & $\begin{array}{l}\text { Adaptarme a una } \\
\text { cultura diferente }\end{array}$ & $\begin{array}{llr}\text { Descubrir } & \text { un nuevo } \\
\text { ambiente } & \text { lleno de } \\
\text { diversidad } & & \end{array}$ \\
\hline España & $\begin{array}{l}\text { Completar mis créditos } \\
\text { de español }\end{array}$ & $\begin{array}{lll}\text { Aprender } & \text { sobre } & \text { la } \\
\text { cultura } & & \end{array}$ & $\begin{array}{l}\text { Experimentar nuevas } \\
\text { cosas }\end{array}$ \\
\hline Costa Rica & $\begin{array}{l}\text { Leer y escribir mejor } \\
\text { en español }\end{array}$ & $\begin{array}{l}\text { Aprender sobre una } \\
\text { nueva cultura }\end{array}$ & $\begin{array}{l}\text { Hablar español } \\
\text { correctamente }\end{array}$ \\
\hline España & Mejorar mi español & $\begin{array}{lr}\text { Aprender } & \text { más } \\
\text { vocabulario } & y \\
\text { gramática } & \end{array}$ & $\begin{array}{l}\text { Relacionarme con los } \\
\text { residentes y aprender su } \\
\text { cultura }\end{array}$ \\
\hline Costa Rica & $\begin{array}{l}\text { Aprender } \\
\text { palabras }\end{array}$ & $\begin{array}{l}\text { Tener la experiencia } \\
\text { de estudiar en el } \\
\text { extranjero }\end{array}$ & \\
\hline
\end{tabular}

${ }^{15}$ Un estudiante no escribió sus expectativas. 


\begin{tabular}{|c|c|c|c|}
\hline \multirow[t]{3}{*}{ España } & Mejorar mi español & Conocer un nuevo & Conocer \\
\hline & escrito & pais hispanohablante & pedagógicos \\
\hline & & & institución universitaria \\
\hline España & Mejorar mi escritura & $\begin{array}{l}\text { Aprender sobre la } \\
\text { cultura en España }\end{array}$ & $\begin{array}{l}\text { Disfrutar y crear buenos } \\
\text { recuerdos }\end{array}$ \\
\hline \multirow[t]{4}{*}{ Costa Rica } & Mejorar $\quad m i$ & Conocer & Mejorar mis acentos \\
\hline & escrito & expresiones & \\
\hline & & $\underline{\text { coloquiales diferentes }}$ & \\
\hline & & a la (sic) que yo utilizo & \\
\hline \multirow[t]{3}{*}{ España } & Mejorar & Aprender sobre & Hacer amigos con gente \\
\hline & vocabulario & diferentes culturas & diferentes \\
\hline & (sic) & & lugares/países \\
\hline \multirow[t]{2}{*}{ España } & Conocer & Divertirme mucho & Ver muchas \\
\hline & personas & & interesantes \\
\hline
\end{tabular}

Es crucial hacer énfasis en que, de las nueve personas que contestaron en este rubro, cuatro de ellas, aproximadamente el $50 \%$, su primera prioridad es "experimentar nuevas cosas, descubrir un nuevo ambiente lleno de diversidad, conocer nuevas personas, relacionarme con los residentes y aprender su cultura"; para dos de ellos constituye la segunda prioridad. Los estudiantes reconocen que, aun siendo hablantes de herencia, están interesados en aprender "otra cultura". ${ }^{16}$ El grupo de estudiantes evaluado corresponde al tipo de estudiantes de herencia que prefiere estudiar en un país diferente al de sus ancestros (Shively, 2016). En ese sentido, al determinar que quieren conocer un ambiente nuevo, relacionarse con los residentes y aprender su cultura, al menos este grupo difiere de la tendencia señalada por Moreno (2009), quien establece en su estudio que la inmersión en

\footnotetext{
${ }^{16}$ En el grupo que fue a Costa Rica en el 2015, de los cuatro estudiantes de herencia, ubicados todos en cursos de gramática de nivel avanzado y superior, tres de los cuatro abandonaron el curso de gramática y se pasaron al de Herencia Cultural Latinoamericana.
} 
otro país no constituye un mayor reto para el $\mathrm{HH}$, puesto que ya se encuentran familiarizados con la cultura y la lengua.

Por otra parte, solo tres determinaron como primera prioridad el querer participar en este tipo de programas para "mejorar mi español escrito". Es importante hacer énfasis en el nivel de conciencia metalingüística de que las respuestas se enfocan en el nivel de lectura y de escritura, estrategias que se aprenden en el contexto escolar y que resulta acorde con el trasfondo educativo mencionado para este grupo (el $70 \%$ no tuvo exposición durante la primaria). Es trascendental que el énfasis sea en los niveles que requieren escolarización, puesto que también indica - por contraposición- que hay conciencia de que se tiene un nivel de lengua oral y de escucha que no requiere instrucción explícita en el aspecto aural, con la única excepción del vocabulario, también mencionado en las expectativas ("mejorar mi vocabulario español", "aprender nuevas palabras") que se adquiere también como parte de la instrucción explícita.

\subsubsection{Participación en el programa con respecto a la lengua española y la cultura}

En relación con la pregunta "mi familia piensa que con respecto a mi conocimiento de español y de la cultura mi participación en este programa es necesaria e importante", las familias en general $(70 \%)$ se manifestaron en completo acuerdo con que el estudiante participara en el PEE. En cuanto a las motivaciones individuales, seis estudiantes estuvieron completamente de acuerdo con que era importante. Tanto las familias como los estudiantes perciben y conceden un valor positivo a la experiencia de estudiar en otro país.

\subsubsection{Desempeño durante los exámenes de ubicación}

En cuanto al desempeño en los exámenes de ubicación, todos los estudiantes reportaron haber completado tanto los exámenes escritos como los orales dentro de los márgenes de tiempo indicado, incluso, como reportó una estudiante, "con tiempo de sobra". Dentro de este núcleo de preguntas sobre el desempeño en los exámenes de ubicación, también se inquirió a los estudiantes sobre algún tipo de dificultad o bien se les pidió que elaboraran sobre algún tipo de retroalimentación que les diera el examinador durante el 
examen oral. Al respecto, se pueden establecer dos tendencias: una valoración positiva del desempeño a nivel oral ("me dijeron que mi examen oral estuvo muy bien", "que hablo muy bien, sin dificultades", "sí, mi español es muy amplio") y valoraciones que revelan aspectos de corrección: "Si, el examinador hizo un comentario acerca de cómo hablo español, ella dijo que tenía que emplear la forma correcta de las palabras y de los verbos en español”.

Otros comentarios revelan un alto nivel de seguridad lingüística en la que el estudiante incluso, en lugar de referirse a lo dicho por el instructor, como se inquiere en la pregunta, responde afirmándose en sí mismo, revela la percepción que tiene sobre él mismo (Cubillos e Ilvento, 2013): "No tuve ninguna. Soy un hablante de herencia, por lo tanto, hablo español de manera fluida y lo entiendo muy bien".

\subsubsection{La experiencia de estudio y cambios en el español}

Los estudiantes también expresaron su valoración de la experiencia en el programa en relación con su español, esto es, aplicaron un proceso de autovaloración (self-efficacy). En su totalidad, expresaron que la vivencia de PEE resultó muy satisfactoria. En cuanto a si la experiencia cambió o no su manera de hablar el español, un 40 \% expresó estar completamente de acuerdo con que su español había cambiado (dos de España, dos de Costa Rica). Al respecto, los estudiantes reportaron: “después de terminar el PEE, me resultó más fácil leer y escribir en español”, “entiendo las distintas palabras de la cultura española comparadas con la cultura mexicana. También mejoré mi escritura y la comprensión de distintas palabras".

Otro $40 \%$ expresó estar más o menos en desacuerdo con la idea de que su español había cambiado (dos estudiantes de España y dos de Costa Rica): “aunque mi español ya es bastante avanzado si estoy de acuerdo que mi estancia en España mejoró mi vocabulario (ya no me trabo tanto cuando hablo, jaja)". Por otro lado, una estudiante (de posgrado) expresó cierta decepción: “Algunas de las destrezas que esperaba mejorar todavía no las he dominado y siento que estas fueron presentadas y evaluadas muy rápidamente. No hubo tiempo para desarrollar la destreza. Sin embargo, siento que mucho del material que fue introducido fue de beneficio". En las dos respuestas se ven percepciones y actitudes basadas en la autopercepción; desde el reconocimiento de tener un nivel avanzado hasta la expectativa no 
cumplida de mejoría. En esa línea, se puede percibir una coincidencia con los resultados del impacto de PEE en estudiantes de español como L2 presentados por Aguilar Stewart (2010) en el sentido de que la experiencia de PEE no rinde los mismos frutos para todos los individuos.

Un $20 \%$ expresó estar en completo desacuerdo, es decir, que sentían que su español no había cambiado (uno de España, uno de Costa Rica); al respecto elaboraron: "No ha cambiado. Hablo igual solamente que mi conocimiento de la lengua ha mejorado". No queda claro si por conocimiento se refiere al uso de la lengua o el conocimiento de la terminología lingüística que se suele emplear en las clases de idioma.

En todos los rubros dentro de esta categoría (valoración sobre el cambio experimentado con respecto al español) se aprecian tendencias parecidas y en las mismas proporciones. Es decir, aquellos que están completamente de acuerdo en que su español cambió, lo expresan tanto en relación con el PEE de España y con el de Costa Rica; aquellos que están más o menos de acuerdo, lo expresan asimismo tanto para España como para Costa Rica de la misma forma que lo manifiestan aquellos que están en completo desacuerdo en que su español haya cambiado. Es decir, que el lugar de destino del PEE (Costa Rica o España) no pareciera ser el elemento determinante en la autopercepción o en la actitud hacia su español.

Al analizar las respuestas del $80 \%$ (completamente de acuerdo, más o menos de acuerdo) se observa que sus valoraciones se refieren a procesos de "entrada y salida" ("input" y “output”) que son aprendidos dentro del ámbito escolar o bien a aspectos de vocabulario. El 20 \% restante establece que "no ha cambiado", es decir, basa su juicio en un aspecto de “ejecución” de la lengua. Al preguntárseles de forma más directa si pensaban que hablaban “mejor" ahora después del PEE, un 70 \% expresó estar completamente de acuerdo/más o menos de acuerdo y un 30 \% expresó estar en completo desacuerdo o le daba igual/no sabía. No obstante, en la última pregunta del cuestionario, al preguntárseles como evaluaban su español después de la experiencia en el PEE, hay un consenso en decir que su español mejoró significativamente ("le daría una A+"), con énfasis, particularmente en la expansión de vocabulario y en la cultura ("mejor, tengo una mejor idea de la cultura de otro país"; "aprendí nuevos términos y conceptos"). 
Otra pregunta del cuestionario inquirió sobre qué tipo de correcciones había realizado el profesor de español y cuál había sido la razón para ello. Varios estudiantes reportaron que se les había corregido "la ortografía y los errores gramaticales en la escritura", "los tensos [tiempos] verbales", “cosas que decimos con mi familia en México pero que no son gramaticalmente correctas como, por ejemplo, yo diría “onde" en lugar de "donde” o "pa que" en lugar de "para que".

A este respecto, una pequeña anécdota experimentada por la autora del artículo puede poner en evidencia las diferencias en percepción entre lo que los estudiantes saben desde un punto de vista performativo de la lengua y lo que se espera que sepan "técnicamente" sobre esta (saber los nombres de los tiempos verbales, por ejemplo). Al final del primer día de clases del programa de verano en Costa Rica, una instructora de español se acercó a la directora del programa (autora) y expresó "Tus estudiantes son muy lindos, pero no saben nada de gramática". Unos momentos después, una de las estudiantes del programa se acercó en busca de ayuda y expresó a la autora: "Profesora, si usted tuviera unos minutos más tarde, quisiera que me explicara el subjuntivo". Al decirle que justo lo acababa de emplear, la estudiante se afirmó en su valoración y contestó que no, que no sabía usar el subjuntivo.

Este es un aspecto de la enseñanza del español a los estudiantes de herencia que refleja una tensión (Holguín Mendoza, en prensa) entre la enseñanza de registros formales y formas empleadas por los estudiantes de herencia que reciben (son calificadas) como formas estigmatizadas, no pertenecientes al registro "formal”. Si bien en este artículo no hay espacio para ahondar en esta tensión, el tema está siendo investigado y trabajado en relación con la enseñanza a HH. ${ }^{17}$ No obstante, la valoración de las habilidades lingüísticas de los estudiantes por parte de la docente y su confirmación por parte de la estudiante (no saben nada de gramática versus quisiera que me explicara el subjuntivo) no revelan la contradicción existente entre la posición prescriptiva de la profesora (saber gramática es igual a dominar términos metalingüísticos) y la competencia comunicativa de la estudiante que no reconoce el subjuntivo, pero lo emplea.

\footnotetext{
${ }^{17}$ Holguín Mendoza, Claudia. (En prensa). Beyond registers of formality and other categories of stigmatization: Style, awareness and agency in SHL education.
} 


\subsubsection{Interacción con miembros de la comunidad meta, percepciones sobre la cultura}

En el rubro en el que se inquirió sobre si hubo dificultades para conversar y compartir con las personas del país, el $70 \%$ de los estudiantes manifestó no haber tenido ningún obstáculo. El 30 \% de los estudiantes que sí manifestaron tener obstáculos recalcaron que se debió a términos diferentes (“Algunas palabras son diferentes en la cultura mexicana con respecto a la cultura española"; "sí, decían palabras con las cuales no estaba familiarizada"); estos estudiantes asistieron al PEE de España. Es decir, que la autoevaluación y percepción de los obstáculos se reducen a una cuestión de vocabulario, particularmente con el español de España. Esta percepción confirma la ya señalada en el estudio de Fernández Moreno (2017) en el que la mayor diferencia percibida por hablantes hispanos de EE. UU. se da en relación con España, es decir, esa "distancia" que lo hace diferente, pero que no lleva al hablante de herencia de EE. UU. a ver su español como un español periférico.

Esta percepción se vio confirmada a través de los diarios digitales y de la reflexión final de curso:

"Mientras he estado aquí en Costa Rica, mi habla no creo que ha mejorado. No porque la universidad no sea buena ni mucho menos, sino que yo siento que mi habla ha mejorado durante los años. Yo voy a México cada año entonces no siento que necesito practicar estando en Costa Rica porque sé la lengua" (diario digital).

"En el tiempo que he estado aquí en Costa Rica yo siento que he mejorado en el conocimiento de las palabras. En (sic) decir yo hablo el español mexicano pero cuando llegue (sic) aquí a Costa Rica aprendí muchas palabras que no se usan en México [...]. Aprendí las palabras de la cultura y del país pero solamente eso fue" (reflexión final).

El aspecto del choque cultural, también evaluado en relación con el impacto que los PEE tienen en los estudiantes, ha sido reportado para los estudiantes de L2 (Shiri, 2013) y se ha percibido como de menor impacto para los hablantes de herencia por su familiaridad con la cultura hispana. No obstante, varios ejemplos de los diarios digitales escritos por los estudiantes apuntan a que el hablante de herencia, sobre todo si va a un país distinto al de sus ancestros (Shively, 2016) también experimenta este tipo de choque cultural. Por ejemplo, con respecto a la comida, un estudiante señaló: 
"Para mí en comparación con la comida mexicana aquí es mucho más común el arroz. En México es el frijol que casi siempre acompaña cualquier plato de comida. En Costa Rica se enfocan mucho en ensaladas con casi cada comida al igual de comer saludable. En mi estilo de comer, no es tanto así [...] y especialmente siendo mexicano. El chile aquí no existe mucho y eso es algo que sí molesta después de pasar tiempo aquí. Un ejemplo es en comer papas, yo estoy impuesto ${ }^{18}$ en ponerle salsa al menos, y también limón dependiendo de las papas. Aquí no hay de la salsa que me gusta para eso y entonces no queda otra opción para comer”.

Con respecto a aspectos de cultura y dinámicas de la vida cotidiana, expresó:

"Algunas de las primeras cosas que vi cuando llegue (sic) a San Jose (sic), Costa Rica era que no usaban direcciones claras. Era algo difícil de entender porque yo estoy tan impuesto de usar direcciones, usar el GPS y llegar a lugares [...] y sin poner atención a donde voy [...]. Pero en Costa Rica no se usa eso, dan direcciones de locaciones populares y de ahí dan distancias métricas [...]. Esto es algo que aunque para mí es extraño, para la gente de Costa Rica es normal, y es parte de su cultura".

Como se puede apreciar, el hablante de herencia también experimenta el tipo de choque cultural que se ha establecido para estudiantes de L2.

\section{Conclusiones}

La investigación de actitudes, percepciones y creencias de los estudiantes de lengua permite establecer resultados sobre la experiencia de aprender otra lengua, a partir de las valoraciones que los estudiantes hacen sobre sí mismos. En el caso de estudiantes de un idioma como segunda lengua, estos resultados se miden en términos del disfrute que produce la experiencia, mejoramiento en la proficiencia así como en notas (Weseley, 2012).

En el caso de los hablantes de herencia examinados en este artículo, algunos de estos resultados coinciden con los reportados por estudiantes de L2, como el de mejoramiento del español. No obstante, otros elementos contribuyen a medir estas actitudes y percepciones en

\footnotetext{
${ }^{18}$ El diccionario de la Real Academia registra "impuesto, -a" como un adjetivo con la marca diatópica para Cuba y México, con el significado de 'acostumbrado'.
} 
el caso de esta población. Uno de estos factores, en el presente estudio, lo constituye la importancia que se le da a la experiencia de estudiar español en otro país, tanto por parte del individuo participante como por la familia.

Un resultado sumamente relevante de la presente investigación es que la mayoría de los estudiantes de herencia poseen expectativas y motivaciones varias para ir a estudiar español a otro país. A diferencia de lo que es percibido por las instituciones que regulan la enseñanza de la lengua (Corros Mazón, 2005), mejorar el español con el objetivo de adquirir un español "estandarizado" no es necesariamente el único o principal motivo que guía a los hablantes de herencia en esta decisión. Los resultados también muestran que los países estudiados no parecieran ser determinantes en la autopercepción y actitud hacia su español.

En general, las actitudes y percepciones de los estudiantes hacia sí mismos revelan que los estudiantes perciben que tienen un conocimiento del español que necesita mejorarse a nivel escrito, pero no a nivel oral. Los resultados de los exámenes orales y las autovaloraciones (soy hablante nativo, soy hablante de herencia y hablo español con fluidez) así lo demuestran. Las valoraciones posteriores al final del programa revelan que, en su percepción, el español de los participantes ha mejorado por la práctica y la exposición, pero no necesariamente que ha cambiado. Algunos, incluso, sienten que no ha mejorado, sino que se ha adaptado (nuevo vocabulario). Las alusiones a la necesidad de corrección, de emplear las palabras y los verbos correctos (como pasó con la retroalimentación en el examen oral) apuntan a que en la enseñanza de español a hablantes de herencia, pesa, aún más que con los hablantes de L2, la idea de que el español debe responder a cierto estándar que ubica a la forma de hablar de estos estudiantes en una posición de subordinación lingüística con respecto a otras variantes, particularmente con aquellos hablantes de variedades que desde las instituciones se valoran como de poco prestigio (Philips, 2015; Clark McEvoy, 2017); de ahí que sea más relevante, también en el contexto de estudios en el extranjero, incorporar a su formación elementos que refuercen e incrementen su conciencia metalingüística y cultural, sociolingüística, desde una perspectiva crítica (Leeman, 2005; Holguín, 2017).

Los estudiantes presentan niveles muy claros de conciencia metalingüística y cultural, lo cual les provee un nivel de gestión y de intervención que indica la importancia de adoptar una nueva posición con respecto a las motivaciones que tienen para participar en un PEE. 
Estos niveles de conciencia metalingüística y cultural que poseen deberían llevarnos a plantear si existe en realidad una necesidad de evaluar la proficiencia de los hablantes de herencia en los mismos términos en que se realiza con los discentes de L2. Es decir, si la evaluación debería realizarse desde parámetros externos al hablante de herencia y su perfil sociolingüístico o desde una perspectiva que tome en cuenta todos estos aspectos de autoevaluación (self-efficacy), particularmente, como en el caso en que se ocupa esta investigación, con participantes que los docentes mismos ubican en niveles avanzados y superiores.

\section{Bibliografía}

Academia Norteamericana de la Lengua Española. Sitio web, www.anle.us. Consultado el 2 de noviembre de 2018.

Aguilar Stewart, Julia. (2010). Using e-journals to Assess Students' Language Awareness and Social Identity During Study Abroad. Foreign Language Annals, 43(1).

Albarracín, Julia. (2016). At the Core and in the Margins. Incorporation of Mexican Immigrants in Two Rural Midwestern Communities. East Lansing: Michigan State University Press

Beaudrie, Sara y Fairclough, Marta. (2012). Spanish as a Heritage Language in the US. The State of the Field. Washington D.C.: Georgetown University Press.

Carrasco, Roberto y Riegelhaupt, Florencia. (2012). META: A model for the continued acquisition of Spanish by Spanish/English Bilinguals in the United States. En Sara Beaudrie y Marta Fairclough (Eds.), Spanish as a Heritage Language in the US (pp. 405-421). The State of the Field. DOI: 10.1080/15235882.2000.10162775

Clark McEvoy, Thomas. (2017). My Spanish is a Jumble: An Examination of Language Ideologies in Teacher with Low Prestige Varieties. Hispanic Studies Review, 2(1)2844.

Corros Mazón, Francisco. (2005). Aspectos pragmáticos, sociolingüísticos e interferencias culturales en la enseñanza de ELE en Estados Unidos. Asociación para la enseñanza del español como lengua extranjera (ASELE), Actas XVI. 
Cubillos, Jorge e Ilvento, Thomas. (2013). The impact of Study Abroad on Students' SelfEfficacy Perceptions. Foreign Language Annals, 45(4), 494-511.

Curso de Herencia Hispana. Programa intensivo de verano. La Casa de las Lenguas. Universidad de Oviedo. Página web http://spanishcourses.uniovi.es. Consultado el 20 de noviembre de 2018.

Davidson, Dan y Lekic, Maria. (2013). The Heritage and Non-Heritage Learner in the Overseas Immersion Context: Comparing Learning Outcomes and Target-Language Utilization in the Russian Flagship. Heritage Language Journal, 10(2), 226-252.

Delany-Barmann, Gloria et al. (inédito). Heritage Learners and Study Abroad: Negotiating Language and Identity. 6th International Workshop on Spanish Sociolinguistics. Tucson, AZ.

Escobar, Anna María y Potowski, Kim. (2015). El español de los Estados Unidos. Cambridge University Press.

Ferguson, Charles. (1959) Diglossia. WORD, 15(2), 325-340. doi: $10.1080 / 00437956.1959 .11659702$

Fundación Universia. http://www.universia.es/universidades. Consultado el 19 de noviembre de 2018.

Holguín Mendoza, Claudia. (En prensa). Beyond registers of formality and other categories of stigmatization: Style, awareness and agency in SHL education.

Holguín Mendoza, Claudia. (2017). Critical Language Awareness (CLA) for Spanish Heritage Language Programs: Implementing a Complete Curriculum. International Multilingual Research Journal. Routledge Taylor \& Francis Group. doi: 10.1080/19313152.2017.1401445

Institutional Research and Planning. Fact book 2017. Western Illinois University. Consultado el 9 de noviembre de 2018.

Jara, Carla. (2006). El español de Costa Rica según los ticos. (Estudios de lingüística popular) (1. a edición). San Pedro: Editorial de la Universidad de Costa Rica. 
Leeman, Jennifer. (2013). Categorizing Latinos in the history of the US Census: The official racialization of Spanish. En J. del Valle (Ed.), A Political History of Spanish: The Making of a Language (pp. 305-324). Cambridge: Cambridge University Press.

Leeman, Jennifer. (2005). Engaging Critical Pedagogy: Spanish for Native Speakers. Foreign Language Annals, 38(1), 35-45.

Institute of International Education. (2017). Top 25 destinations of U.S. Study Abroad Students 2014/15-2015/16. Open Doors Report on International Educational Exchange. Recuperado de http://www.iie.org/opendoors. Consultado en línea el 28 de septiembre de 2018.

Institute for International Education. (2017). Profile of US Study Abroad Students, 2004/052015/16. Open Doors Report on International Educational Exchange. Recuperado de http://www.iie.org/opendoors

Instituto Cervantes. Sección Estudia Español en España. Página web www.eee.cervantes.es. Consultado el 19 de noviembre de 2018.

Instituto Nacional de Estadística, adscrito al Ministerio de Economía y Empresa de España. Página web $\underline{\text { www.ine.es. }}$ Consultado el 19 de noviembre de 2018.

Instituto Nacional de Estadística y Censos. República de Costa Rica. Página web www.inec.go.cr. Consultado el 19 de noviembre de 2018.

Language International (Portal). Página web www.languageinternational.mx. Consultado el 20 de noviembre de 2018.

Mar-Molinero, Clare y Paffey, Darren. (2011). Linguistic Imperialism: Who Owns Global Spanish? En Manuel Díaz-Campos (Ed.), The Handbook of Spanish Sociolinguistics (pp. 748-763). John Wiley Sons.

Marijuan, Silvia y Sanz, Cristina. (2018). Expanding boundaries: Current and New Directions in Study Abroad Research and Practice. Foreign Language Annals, 51(1), 185-204.

Martínez, Glenn. (2006). Mexican Americans and Language. Del dicho al hecho. Tucson: The University of Arizona Press. 
Mejías, Hugo y Anderson-Mejías, Pamela. (2003). Attitude update: Spanish in the South Texas Border. Hispania, 86(1), 138-150.

Moreno Fernández, Francisco. (2017). Cómo es percibido el español de los Estados Unidos. En Rosa Tezano-Pinto (Ed.), La presencia hispana y el español de los Estados Unidos. Unidad en la diversidad. I Congreso de la Academia Norteamericana de la Lengua. 6-8 de junio de 2014.

Moreno, K.H. (2009). The study abroad experiencies of heritage language learners: Discourses of identity (Tesis doctoral). Universidad de Texas, Austin.

Philips, Susan. (2015). Language ideologies. En Deborah Tannen, Heidi Hamilton y Deborah Schiffrin (Eds.), The Handbook of Discourse Analysis (2nd edition) (pp. 557-575). John Wiley.

Preston, Denison. (2004). Language with an Attitude. En J. K. Chambers, Peter Trudgill y Natalie Schilling-Estes (Eds.), The Handbook of Language Variation and Change (2nd edition). Blackwell Publishing.

Russel, Britanny. (2015). High School Spanish Teacher's Attitudes and Practices Toward Spanish Heritage Language Learners. Foreign Language Annals, 48(3), 413-433.

Said-Mohan, Aixa. (2011). The teaching of Spanish as a Heritage Language: Overview of what we Need to Know as Educators. Porta Linguarum, (16), 89-104.

Shiri, Sonia. (2013). Learners' Attitudes Toward Regional Dialects and Destination Preferences in Study Abroad. Foreign Language Annals, 46, 565-587. doi: 10.1111/flan. 12058

Swender, Elvira, Martin, Cynthia et al. (2014). Exploring Oral Proficiency Profiles of Heritage Speakers of Russian and Spanish. Foreign Language Annals, 47(3), 423446.

United States Census Bureau. (2017). Annual Estimates of the Resident Population by Sex, Age, Race, and Hispanic Origin for the United States and States: April 1, 2010 to July 1, 2016. Recuperado de https://www.census.gov/newsroom/facts-forfeatures/2017/hispanic-heritage.html 
Universidad de Costa Rica (Portal). www.universidades.cr. Consultado el 19 de noviembre de 2018.

Valdés, Guadalupe. (2001). Heritage language students: Profiles and Possibilities. En Joy Peytton, Donald Ranard y Scott McGinnis (Eds.), Heritage Languages in America: Preserving a National Resource. Language in Education: Theory and Practice (pp. 1-41). Washington D.C.: Center for Applied Linguistics (CAL).

Valdés, Guadalupe et al. (2003). Language ideology: The Case of Spanish in Departments of Foreign Languages. Anthropology and Education Quaterly, 34, 3-26.

Villa, Daniel. (1996). Choosing a "Standard" Variety of Spanish for the Instruction of Native Speakers in the US. Foreign Language Annals, 29(2), 191-200.

Villa, Daniel. (2002). The sanitizing of U.S. Spanish in Academia. Foreign Language Annals, 35(2), 222-230.

Wesely, Pamela. (2012). Learner Attitudes, Perceptions, and Beliefs in Language Learning. Foreign Language Annals, 45(1), s98-s117. doi: 10.111/j.1944-9720.2012.01181.x 


\section{Anexos}

Anexo 1. Cuestionario de expectativas y metas para hacer un programa de estudios en el extranjero

1. ¿En qué país realizó su programa en el extranjero?
a. Costa Rica
b. España

2. Mi motivación para hacer un programa de estudios en el extranjero es porque (marque todas las que aplican):
a. Necesito los créditos de los cursos para mi especialidad en español
b. Para graduarme
c. Para mejorar mi español
d. Para conocer un país de habla hispana

3. Con este programa de estudios en el extranjero tengo/tuve las siguientes expectativas:

4. Ordene sus expectativas en orden de prioridad (por ejemplo 2,1,3).

5. Sobre mi participación en este programa, mi familia piensa que con respecto a mi conocimiento de español y de la cultura, mi participación en este programa es necesario e importante:
a. Completamente en desacuerdo
b. Más o menos en desacuerdo
c. Les da igual/no saben
d. Más o menos de acuerdo
e. Completamente de acuerdo

6. Sobre mi participación en este programa, yo pienso que con respecto a mi conocimiento de español y de la cultura, mi participación en este programa es necesaria e importante:
a. Completamente en desacuerdo
b. Más o menos en desacuerdo
c. Les da igual/no saben
d. Más o menos de acuerdo
e. Completamente de acuerdo 
7. ¿Pudo usted completar su examen de ubicación (placement test) escrito?

8. ¿Pudo usted realizar su examen de ubicación de conversación sin dificultades?

9. ¿Experimentó usted algún tipo de dificultad comunicativa con su examinador durante el examen oral? Comente.

10. ¿Recibió usted algún tipo de comentario/retroalimentación sobre comprensión su examen oral?

11. Para este programa, ¿en qué nivel fue ubicado después de su examen de ubicación?
a. Básico 1 o inicial
b. Básico 2 o inicial avanzado
c. Intermedio $1 \mathrm{o}$ intermedio inicial
d. Intermedio 2 o intermedio avanzado
e. Avanzado 1 o avanzado inicial
f. Avanzado 2 o avanzado intermedio
g. Superior (otro curso más avanzado o curso electivo). ¿Cuál?

12. Durante el examen oral, entender a su entrevistador fue
a. Fácil
b. Más o menos fácil
c. Normal
d. Difícil
e. Bastante difícil

13. Explique:

14. ¿Cómo califica usted su experiencia en el programa en relación con su español?
a. Muy satisfactoria
b. Bastante satisfactoria
c. Satisfactoria
d. No muy satisfactoria
e. No me agradó

15. ¿Siente que su español ha cambiado en algún sentido

a. Completamente en desacuerdo 
b. Más o menos en desacuerdo

c. Le da igual/no sabe

d. Más o menos de acuerdo

e. Completamente de acuerdo

16. Elabore

17. Durante sus clases durante el programa, ¿qué cosas aprendió que no sabía antes?

18. Durante sus clases en el programa, ¿hubo algún aspecto del lenguaje que su instructor le corrigió o pidió que cambiara? Explique.

19. ¿Por qué le pidió su instructor que lo cambiara? ¿Cuál fue el comentario?

20. ¿Usted piensa que puede usar esas nuevas ideas o conceptos, palabras o expresiones, con su familia de regreso en los Estados Unidos?

21. ¿Siente que habla "mejor" ahora?
a. Completamente en desacuerdo
b. Más o menos en desacuerdo
c. Me da igual/no sé
d. Más o menos de acuerdo
e. Completamente de acuerdo

22. En relación con el español que se habla en el país donde hizo el programa, ¿tuvo dificultades para conversar y compartir con las personas del país?
a. Sí
b. No

23. ¿Qué tipo de dificultades tuvo, si es que las tuvo? Comente.

24. ¿Cómo evalúa su español después de la experiencia de este programa?

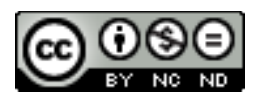

Esta obra está bajo una licencia de Creative Commons Reconocimiento-NoComercial$\underline{\text { SinObraDerivada } 4.0 \text { Internacional }}$ 